\title{
Relative Stabilität: Winkelstabilität am distalen Radius, die LCP-T-Platte 3.5
}

\author{
Irene Jantzen
}

\section{Zusammenfassung}

Die winkelstabile Verplattung der distalen Radiusfraktur kann mit Implantaten der Größe 2.0, 2.4 oder 3.5 durchgeführt werden. Bei hoher Stabilität der Implantate durch die gleichmäßige Verteilung der Kräfte auf alle kopfverriegelten Schrauben geschieht die Heilung der Fraktur nach den Prinzipien der relativen Stabilität auf dem Wege der sekundären Knochenbruchheilung, die zu ausgeprägter Kallusbildung führt.
Relative Stability: Angular Stability at the Distal Radius, the LCP-T Plate 3.5

Angle-stable fixation of distal radius fractures can be achieved with implants of the size $2.0,2.4$ or 3.5. With the high stability of the implant due to the uniform distribution of forces to all the head-locked screws, healing of the fracture occurs according to the principles of relative stability on the way to secondary fracture healing which leads to pronounced formation of callus.

\section{Lernziele}

1. Was bedeutet Winkelstabilität für die Frakturheilung?

2. Welche Vorteile hat Winkelstabilität am distalen Radius?

3. Implantate

Ziel jeder operativen Frakturbehandlung ist die Wiederherstellung der früheren, vollen Funktion der verletzten Extremität. Wird eine Fraktur so stabilisiert, dass im Frakturbereich unter dynamischer und funktioneller Beanspruchung noch minimale Bewegung stattfinden kann, erfolgt die Heilung der Fraktur über die Bildung von Kallus. Man spricht von sekundärer Knochenbruchheilung durch relative Stabilität.

\section{Was bedeutet die feste Verbindung von Schraube und Platte bei der Kopfverriegelungstechnik?}

Die Kraftübertragung zwischen den beiden Knochenfragmenten geht durch die kalt verschweißte Verbindung zwischen Kopfverriegelungsschraube und Platte fast ohne Verlust, obwohl die Frakturenden nicht aufeinandergepresst werden (Abb.1).

Die beiden Vorteile der primären und der sekundären Knochenbruchheilung verbinden sich: Wir erreichen eine besonders hohe Stabilität des Implantates bei guter Kallusbildung. Das Periost und die Durchblutung der Knochenfragmente werden geschont („a balance between stability and biological integrity“).
Zudem werden durch die gleichmäßige Verteilung des Druckes auf alle kopfverriegelten Schrauben diese vor allem im osteoporotischen Knochen besser verankert. Dies führt nicht nur zu einer verbesserten Langzeitstabilität, sondern auch zu weniger Implantatversagen.

\section{Was bedeutet dies für die Fraktur- heilung?}

- Hohe Stabilität der Fraktur

- Kallusbildung durch sekundäre Knochenbruchheilung

Am distalen Radius werden Implantate der Stärken 2.0, 2.4 und 3.5 verwendet zunehmend winkelstabil.

Indikationen für eine LCP-3.5-T-Platte am distalen Radius sind metaphysäre Frakturen, aber auch Frakturen mit Gelenkbeteiligung.

\section{Implantate}

Die winkelstabilen T-Platten mit 3-5 Löchern im Schaft dienen zur palmaren, die mit schrägem Querschenkel für rechts und links zur dorsalen Stabilisierung (Abb. 2). Es liegt jedoch im Ermessen des Operateurs, sie auch anders zu verwenden.

Es können Kopfverriegelungsschrauben, aber auch Standard-Kleinfragmentschrauben verwendet werden (Abb.3), jedoch sollten zumindest alle Schrauben des Querschenkels mit winkelstabilen Schrauben besetzt sein, sofern es die Fraktur erlaubt. 


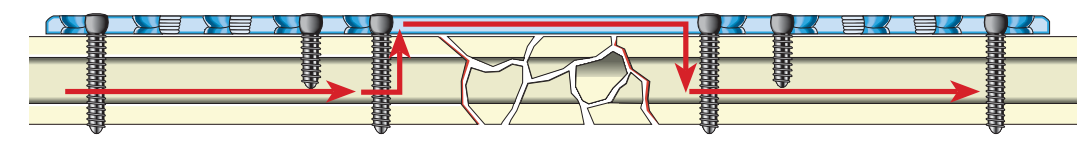

Abb. 1 Winkelstabilität (nach: Wagner M, Frigg R. AO Manual of Fracture Management, Internal Fixators, Concepts and Cases using LCP and LISS. New York: Thieme, 2006, S. 22 [1]).

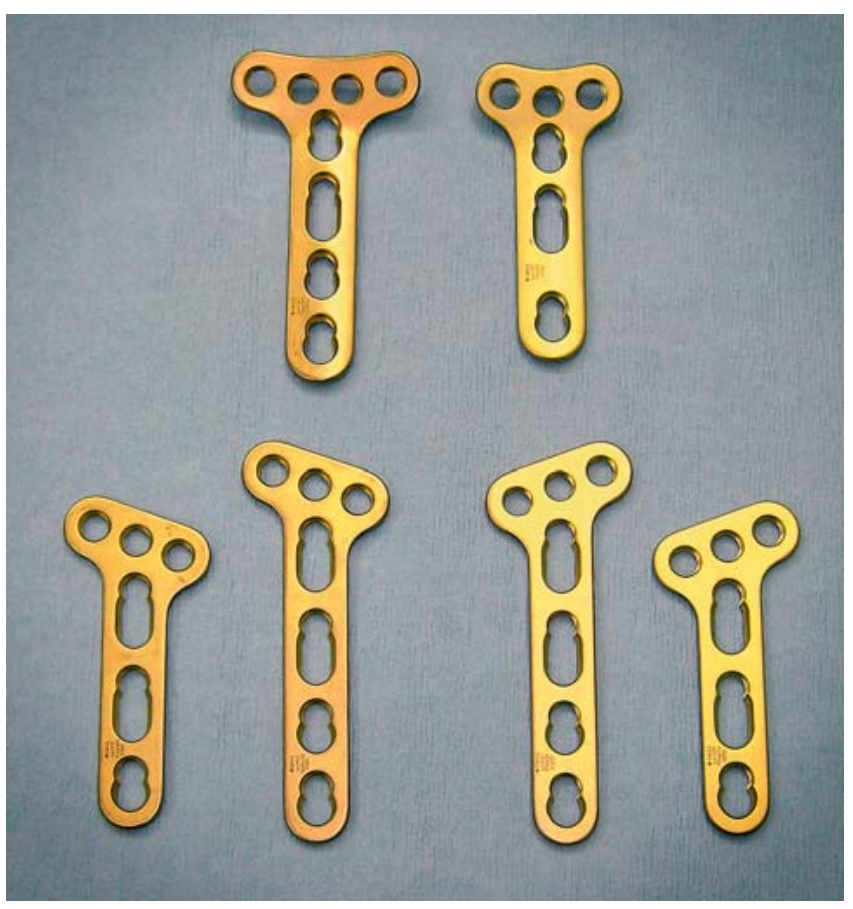

Abb. 2 Platten. 441.131: T-Platte mit 3 Löchern im Schaft; 441.141: T-Platte mit 4 Löchern im Schaft; 441.931: schrägwinklige T-Platte mit 3 Löchern im Schaft, links; 441.941: s. o. mit 4 Löchern im Schaft; 441.031: schrägwinklige T-Platte rechts mit 3 Löchern im Schaft; 441.041: s. o. mit 4 Löchern im Schaft.

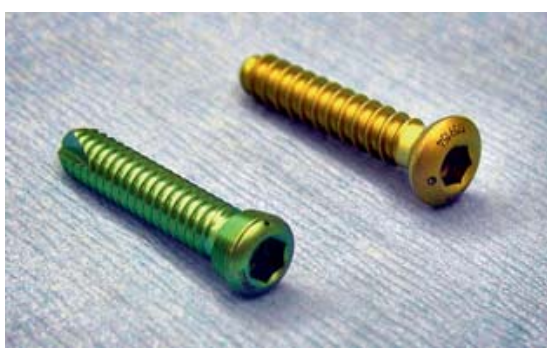

Abb. 3 Schrauben. 413.020: LCP-Verriegelungsschraube $3,5 \mathrm{~mm}$, selbstschneidend, Titan, $20 \mathrm{~mm}$; 404.020: Kortikalisschraube, $3,5 \mathrm{~mm}$, Titan, $20 \mathrm{~mm}$.

\section{Vorgehen}

Die zu operierende Seite wird steril abgewaschen und beweglich bis Mitte Oberarm abgedeckt. Bei geschlossener Blutsperre: Hautschnitt und Freipräparieren der Fraktur. Nach Reposition unter Bildwandlerkontrolle wird die Platte eingebracht und mit einer nicht winkelstabilen Kortikalisschraube in einem der länglichen Schaftlöcher vorläufig fixiert. Wir brauchen (Abb. 4):

- Spiralbohrer 2,5 mm

- Universalbohrbüchse

- Messgerät

- Gewindeschneider für Kortikalisschrauben $3,5 \mathrm{~mm}$

- Sechskantschraubenzieher klein

Abb. 5 LCP-Instrumentarium. 323.027: LCP-Bohrbüchse 3.5; 310.284: LCP-Spiralbohrer 2,8 mm; Drehmomentbegrenzer (511.770) mit Handgriff (397.705) und Sechskantschraubenzieher-Einsatz klein (314.030).

Abb. 4 Kleinfragmentinstrumentarium. 310.250: Spiralbohrer 2,5 mm; 323.360: Universalbohrbüchse 3.5; 319.010: Tiefenmessgerät; 311.320: Gewindeschneider für Kortikalisschrauben $3,5 \mathrm{~mm}$; 314.070: Sechskantschraubenzieher klein, 2,5 mm. 
Bei leicht gelöster Kortikalschraube kann die Platte unter Bildwandlerkontrolle richtig platziert werden. Die Schrauben des Querschenkels werden nun winkelstabil besetzt.

Wir brauchen (Abb. 5):

- Winkelstabile LCP-Bohrbüchse 3,5 $\mathrm{mm}$

- Kalibrierter LCP-Spiralbohrer 2,8 mm

- Drehmomentbegrenzer mit Handgriff und Sechskantschraubenzieher-Einsatz klein

Nach erneuter Bildwandlerkontrolle können nun die Schaftschrauben wahlweise winkelstabil oder herkömmlich besetzt werden. Ist die abschließende Bildwandlerkontrolle zufriedenstellend, werden palmar der Musculus pronator quadratus, dorsal das Retinaculum ex- tensorum über der Platte adaptiert. Zur Hämatomableitung empfiehlt sich das Einbringen einer Redon-Drainage vor dem Adaptieren des subkutanen Gewebes. Es folgen Abschlussdesinfektion, Hautnaht und Anlegen eines sterilen Verbandes.

\section{Nachbehandlung}

Bei Übungsstabilität der versorgten Fraktur wird eine Gipsschiene für 6 Wochen angelegt und gleich mit Physiotherapie begonnen. Handelt es sich um eine Trümmerfraktur, bei der keine Übungsstabilität erreicht werden konnte, wird das Handgelenk vor dieser Weiterbehandlung noch für ein bis zwei Wochen in einem zirkulären Gips ruhig gestellt.

\section{Literatur}

1 Wagner M, Frigg R. AO Manual of Fracture Management, Internal Fixators, Concepts and Cases using LCP and LISS. New York: Thieme, 2006

2 Rüedi TP, Murphy WM. AO Principles of Fracture Management. New York: Thieme, 2000

\section{Irene Jantzen}

Fachschwester für den

Operationsdienst

Berufsgenossenschaftliche Unfallklinik Tübingen

Schnarrenbergstraße 95

72076 Tübingen

E-Mail: nenajantzen@web.de 